\title{
Saberes científicos y regímenes violentos: confluencia de ideas, prácticas e intereses entre la psiquiatría alemana y el régimen nazi.
}

Resumen: El artículo que sigue se interesa por el vínculo gestado entre el régimen nazi y la disciplina psiquiátrica alemana, con el objeto de poner en tensión ciertas prácticas y conceptualizaciones suscitadas en la comunidad médica, las influencias culturales y racistas del espacio alemán en las mismas, así como su participación en los programas del nazismo en el poder. El objetivo central del trabajo es reconstruir, a partir de producciones historiográficas contemporáneas, un proceso descuidado, referido a las muertes que el Estado nazi perpetró sobre un conjunto extenso de sujetos calificados como peligrosos y degenerativos de la raza, entre los cuales se encontraban los mismos alemanes. Se propone, por otra parte, reafirmar una postura que entienda al ascenso del nazismo como un proceso complejo, atravesado fundamentalmente por factores culturales, intelectuales y socio-económicos, entre los cuales la política resultó un medio para canalizarlos, antes que una vía explicatoria. Para ello, en primer término desarrollaremos algunas líneas del panorama sociopolítico y cultural de la Alemania previa al ascenso del nazismo, para de esa manera poder concebir no sólo la posibilidad de ascenso del régimen, sino también la emergencia, desde principios de siglo, de corrientes intelectuales con un fuerte contenido racial en el pensamiento psiquiátrico, que luego resultaron afines y justificatorias del régimen. Avanzaremos, en segundo lugar, sobre las principales instancias en que dichos saberes científicamente legitimados se articularon con el Tercer Reich desde la academia, las corporaciones profesionales y el mundo industrial.

Palabras clave: Historia de la Psiquiatría; Profesionalización; Nazismo

Abstract: The following article is interested in the link gestated between the Nazi regime and the German psychiatric discipline, in order to question certain practices and concepts raised in the medical community, its cultural and racial influences in

\footnotetext{
* Consejo Nacional de Investigaciones Científicas y Técnicas (Argentina) e Facultad de Psicología, Universidad Nacional de Rosario (Argentina) Profesor de Teoría Social en la Facultad de Psicología de la Universidad Nacional de Rosario.
} 


\section{Revista Brasileira de História \& Ciências Sociais - RBHCS}

Vol. $8 \mathrm{~N}^{\mathrm{o}}$ 16, Julho - Dezembro de 2016

the German space, as well as their participation in the programs of Nazism in power. The central objective of this work is to reconstruct, from contemporary historiographical productions, a disregarded process, referred to the deaths the Nazi state perpetrated on a vast set of subjects qualified as dangerous and race degenerative, among which we could find Germans themselves. It intends, also, to reaffirm a position that understands the rise of Nazism as a complex process, mainly crossed by socioeconomic, cultural, intellectual factors, where politics were a means to canalize them, rather than an explanatory way. To pursue this, we will first develop some lines of socio-political and cultural landscape of Germany previous the rise of Nazism, to thereby be able to see not only the rising possibility of the regime, but also the emergence, since the beginning of the century, of intellectual currents with a strong racial content on psychiatric thought, which then were related to the regime and act as it justification. We advance, secondly, on the main instances in which such knowledge scientifically legitimated was articulated with the Third Reich from the academia, the professional corporations and the industrial world.

Key words: History of Psychiatry; Profesionalization; Nazism

\section{Introducción: la construcción de Alemania, cambio de siglo y contexto internacional}

El abordaje de la otredad efectuada por el Nazismo, en una radicalidad excluyente que llegó al exterminio planificado de poblaciones en su territorio y en los distintos países que anexó, constituye un tópico historiográfico de peso, y ha sido interrogado ya desde numerosas facetas, ${ }^{1}$ aunque no es este el objeto que nos convoca. Dada la magnitud del genocidio perpetrado contra el pueblo judío y otras "minorías" -como gitanos y homosexuales, entre otros-, la lente del historiador y la recuperación de la memoria colectiva se han centrado mayormente en estos hechos, de innegable relevancia. Sin embargo, no fueron éstos los únicos asesinatos perpetrados. La política de exterminio de un autre peligroso no se resumió en los enemigos construidos como "externos" al ascenso del régimen, y en este sentido, tanto la

\footnotetext{
1 En esta dirección, Enzo Traverso ha revisitado los principales debates en torno a la historización del nazismo como proceso socio-político concreto y signado por la violencia, así como tambien en el plano de los Holocaust Studies, sobre las posibilidades de concebir y estudiar la Shoa en tanto genocidio (TRAVERSO, 2012; 2004).
} 
historia cultural como la historia de la ciencia han permitido tomar dimensión de una serie de procesos y tendencias que dan cuenta de la magnitud del programa de transformación racial encarnado en el Estado, el cual llegó a dirigirse contra los propios alemanes. En el artículo que sigue nos ocuparemos del tratamiento historiográfico de esta zona gris de dicho proyecto eugenésico, en la cual se entramaron el ascenso del régimen nazi al poder y la disciplina psiquiátrica alemana, y que puede pensarse, en parte, en la confluencia de múltiples tendencias que anteceden al período iniciado en 1933.

Aquello que a fines de la Gran Guerra se conocía por Alemania -de manera análoga para el caso italiano- era en verdad el fruto de una invención geopolítica muy particular que unificó hacia 1870 un conjunto de reinos germánicos con un fuerte contenido nacionalista anclado en el volk, bajo la gestión del canciller Bismark, primer ministro de Prusia desde 1862. Los avatares que estas minorías atravesaron desde mediados del siglo XIX en su vínculo con el Imperio Austro-Húngaro signaron el contenido agresivo en su concepción de "lo nacional", situación que marcó la pauta del régimen que desde la década del setenta se llevó a cabo. La unificación efectiva de las "alemanias" realmente existentes puede comprenderse a partir de tres medios implementados por Bismarck: la industrialización acelerada de Prusia, una política internacional agresiva -para liberarse del yugo de Austria- y un sistema político que, si bien constitucional y democrático, sostenía un fuerte cariz autoritario al ser en extremo personalista -primero para con el emperador y luego hacia el canciller-, y delegar demasiados poderes del Reichstag en el primer ministro, al tiempo que desconectaba la vida política del ejercicio del gobierno por el lugar atribuido a las elites burocráticas (CASALI DE BABOT; PRIVITELLO, 2001).

Con el final de la Primer Guerra, en la cual Alemania experimentó la mayor derrota, se desataron tres procesos que modificaron rotundamente el contexto europeo. Por una parte, se disolvieron las tres formaciones imperiales más extensas -Austrohúngara, Rusa y Otomana-; se reconfiguró, así, el mapa geopolítico de Europa y, por último, se firmó el Tratado de Versalles en 1919, que selló el devenir conflictivo y violento de las relaciones internacionales en menos de una década con sus imposiciones sobre los vencidos y los contextos nacionales existentes. En este escenario, Alemania acusó recibo de dichas transformaciones de manera abrupta. Por un parte, por la instauración forzada de gobierno republicano y constitucional cuya inviabilidad estaba signada de antemano, no sólo por las características que el 
ejercicio de la política y el poder político tenían en suelo germano, sino también por un contexto europeo de entreguerras en el cual el liberalismo y sus formas políticas de gobierno se encontraban fuertemente cuestionados -uno más de los corolarios de la Gran Guerra-. ${ }^{2}$ En segundo término, la economía alemana adoptó una efervescencia artificial durante los años veinte, mientras que la economía mundial no disponía de tal dinamismo, puesto que su base se constituía de préstamos internacionales y apropiación de ámbitos productivos en otros países, lo cual explica su fragilidad. Paradojal, aunque inevitable, fue el proceso inflacionario desatado consecuencia directa de Versalles- en el marco del cual la aplicación del Plan Dawes consolidó los efectos sociales de la inflación, favoreciendo la confiscación y transferencia de ingresos hacia ciertos sectores, así como la concentración industrial y bancaria (FANO, 1985).

Por último, el Estado alemán -y su sistema político- vio limitadas sus funciones de forma considerable en el plano material, tanto por el crecimiento del paro y la consecuente debilidad fiscal -en especial desde la crisis de 1929-, como por el sostenido avance del nazismo como fuerza política. 3 La inestabilidad política de los primeros tres años de la década del treinta resultó una escalada donde se articularon problemas coyunturales, tensiones gestadas décadas atrás y la creciente aceptación del lenguaje de la violencia como práctica política legítima (KERSHAW, 1989). En efecto, en 1930, el Partido Nazi pasa de obtener 800.000 votos a casi 6.5 millones, incrementando sus escaños parlamentarios de 12 a 107, mientras que en 1931 crearon un frente nacional contra el bolchevismo, con lo cual ampliaron su captación de los sectores de derecha, sumado ello al accionar de las Sturmabteilung y luego las Schutzstaffel -las SA y SS, respectivamente-, fuerzas paramilitares del partido. En

$2 \mathrm{El}$ problema de la legitimidad y eficacia del Estado y del sistema político como tal fue un tropos candente en la entreguerras. En su evaluación deben ponderarse no sólo el impacto de la Primera Guerra, sino también la emergencia de otros modelos alternativos de sociedad. Así, el lugar del líder adquiere otra tónica en el marco de la crisis generalizada, y más aún cuando se carecía de consensos básicos sobre el rol del Estado como representante de los intereses de la nación. En este sentido, el juego de fuerzas socio-políticas operaban en el sistema, pero en contra de sus reglas, y el problema fundamental, sostiene Juan Linz, radica en la legitimidad, que se agrava ante la imposibilidad de resolver los problemas que se suscitaban (LINZ, 1991).

3 Por otro lado, debemos inscribir este acrecimiento en el marco del progresivo avance de las derechas, reaccionarias, defensivas, nacionalistas y opuestas a los valores e instituciones del liberalismo. Hobsbawm entendió que el ascenso de estas fuerzas respondía al temor por tres realidades concretas: por un lado, el fortalecimiento de los movimientos obreros organizados que ponían en jaque el orden establecido; por otro, el devenir del bolchevismo y, por último, el desencanto provocado por la Primera Guerra (HOBSBAWM, 1995). Otras lecturas sobre este ascenso, enfatizando cuestiones relativas a la imagen "optimista" de la propuesta del partido en un escenario social cargado de opacidad, junto a un análisis de una nueva forma de configurar las identidades políticas entre 1914 y 1933 (FRITZSCHE, 2009). 
esta dinámica, el ascenso al poder de Hitler se operó no sólo por vías legales, sino también como parte de las tensiones experimentadas al interior de la coalición socialdemócrata, así como por la existencia de una laguna autoritaria de la Constitución de Weimar, dada de baja por Hitler una vez que accedió al puesto de canciller, el 30 de enero de 1933 (GAY, 2011).

\section{II -Discursos, ideas y representaciones: salud pública, medicina y autonomización de la Psiquiatría}

Para poder comprender el lugar del saber psiquiátrico en su articulación con el nazismo entendemos que es preciso, en primer término, fijar nuestra atención sobre los intrincados lazos que en Alemania unían la academia, la política, la industria y las fuerzas armadas. En este sentido, partimos de la premisa de que por encima de los vínculos concretos y rastreables que puedan señalarse entre los científicos, las corporaciones y el régimen, va de suyo que la circulación de ideas y discursos en una sociedad -en especial aquellos legitimados por una verdad científica4-, dan lugar a cierta configuración de las representaciones y las sensibilidades en cada período5. El hecho de que en el campo médico hayan llegado a desarrollar mecanismos y técnicas de exterminio de poblaciones consideradas inferiores no fue un corolario del nazismo, sin más bien la expresión de que en el campo representacional y axiológico del volkish germano existían las condiciones de posibilidad que permitieron concebir dicho proyecto. Rastrear estos mojones en el campo de las ideas médicas es un paso ineludible en esta dirección.

El pensamiento germano fue prolífico en una miríada de ámbitos del saber, y la medicina no estuvo exenta. En este trabajo rescataremos dos componentes de dicho devenir intelectual. Por una parte, la tesitura que adoptó el nacionalismo alemán, en

\footnotetext{
4 No es preciso aclarar que un enunciado de cualquier tipo no posee el mismo estatuto epistemológico si es la actividad científica la que lo sustenta. No es menor conservar, empero, una actitud crítica en este punto. La colonización de la vida a partir de la construcción de regímenes de veridicción y subjetivación históricos y específicos merece fue interpelada por Michel Foucault en numerosas de sus obras. Dados nuestros intereses, recuperamos dicha problematización a partir de los cursos en el Collége de France sobre El poder psiquiátrico, Los Anormales y Defender la sociedad (FOUCAULT, 2012 [2003]; 2010 [1999]).

5 En este punto seguimos a Marc Angenot, quien conceptualizó la posibilidad misma del pensar y decir en una sociedad a partir de la circulación de ideas y discursos bajo el concepto de discurso social, por el que entiende "los sistemas genéricos, los repertorios tópicos, las reglas d encadenamiento de enunciados que, en una sociedad dada, organizan lo decible -lo narrable y opinable- y aseguran la división del trabajo discursivo. Se trata entonces de hacer aparecer un sistema regulador global cuya naturaleza no se ofrece inmediatamente a la observación, reglas de producción y circulación, así como un cuadro e productos." (ANGENOT, 2010, p. 21-22).
} 


\section{Revista Brasileira de História \& Ciências Sociais - RBHCS}

Vol. $8 \mathrm{~N}^{\mathrm{o}}$ 16, Julho - Dezembro de 2016

lo que se definió contemporáneamente como modernismo reaccionario. Este resultó de la confluencia del Romanticismo alemán y el volkisch, junto con un anticapitalismo selectivo y una recepción irracionalista de la tecnología, configurando, en igual medida, un campo de exclusión biológica con los semitas como enemigos públicos y raciales. 6

En segundo lugar, desde mediados del siglo XVII, el desarrollo de la práctica médica y la prestación de servicios de salud se perfiló como "ciencia del Estado" (Staatswissenchaft). Ello fue posible a partir de dos procesos correlativos: en primer término, el avance de la medicina en su apelación al Estado, tomándolo como objeto e instrumento de acción con miras a optimizar los reducidos recursos con que contaban estos reinos germanos. En segundo lugar, además, el Estado mismo se constituyó en el espacio de confluencia, ocupación y apelación corporativa de un proceso de normalización de la práctica, el saber y la profesión médica, a partir de lo cual dio lugar a la emergencia de una medicina de Estado que concebía la atención de la salud en relación a los diversos conflictos que el Estado debía afrontar, procurando preservar la fuerza de sus miembros como modo de fortalecerlo (FOUCAULT, 1996).

En esta dirección, la tradición médica alemana sustentaba su praxis en un enfoque holístico (Ganzheitslehre), que hasta el primer cuarto del siglo XX fue hegemonizado por una concepción del cuidado del Otro pensada en términos individuales (Fürsorge). Dicho enfoque continuó y se reforzó durante la República de Weimar, donde la salud individual de cada sujeto procuraba atenderse en forma integral (SEEMAN, 2005). La Psiquiatría alemana, por su parte, se distancia durante el siglo XIX de la escuela francesa -en la que primaban las aproximaciones morales-, hacia un enfoque de tipo somático. Esto respondía a un proceso de más amplio alcance de autonomización y profesionalización de la Psiquiatría como disciplina respecto a la medicina general durante el último tercio del siglo XIX, en donde los psiquiatras se esforzaron considerablemente por delimitar su área de expertise. En esta transición, que condujo del alienismo a una psiquiatría con su propio estatuto científico, fueron fundamentales la construcción de nosologías y el desarrollo de un nuevo anclaje sobre los factores biológicos de cada enfermedad, así como el establecimiento de

6 En su excepcional estudio al respecto, Jeffrey Herf afirma que la tradición modernista reaccionaria era políticamente congruente en tres sentidos: contribuía al vigor tecnológico que hacía a la guerra concebible; preservaba un ethos anticientífico y antirracional que bloqueaba toda adopción de las innovaciones técnicas de otros países, y, por último, formaba parte del fanatismo ideológico que convenció a los nazis que podían ganar. Su mayor problema, sin embargo, fue que sustituyó una coordinación estratégica de los fines por una apuesta política (HERF, 1990). 


\section{Revista Brasileira de História \& Ciências Sociais - RBHCS}

Vol. $8 \mathrm{~N}^{\mathrm{o}}$ 16, Julho - Dezembro de 2016

distancias respecto a los tempranos desarrollos del psicoanálisis, siendo recién hacia la década de 1920 cuando algunos médicos comienzan a incursionar en estas lecturas y sus cruces, aunque estaban mucho más vinculados con instituciones austríacas y suizas que alemanas (ROELCKE, 2005). Esta somatización del saber psiquiátrico fue posible, asimismo, gracias a la circulación y recepción de otras corrientes intelectuales como la criminología positivista italiana y la teoría de la degeneración, así como por el avance de una concepción anatomo-clínica en los estudios médicos, cuyo mayor ejemplo se observa en los neurólogos y neuropatólogos alemanes (LÓPEZ-MUÑOZ et al, 2008).7

La construcción de patologías biologizadas y medicalizables fue el área donde los alemanes señalaron su mayor distancia respecto a la tradición francesa, adoptando activamente métodos y prácticas de las ciencias naturales, la estadística y las "ciencias sociales" de su tiempo. En esta dirección, avanzaron delimitando de forma progresiva saberes, instituciones, y esferas de intervención social específicas, así como también la extensión de su injerencia respecto a sus ámbitos tradicionales, como el hospital o la academia. Fueron cruciales en este proceso la gesta de asociaciones profesionales, además de la incorporación del diagnóstico psiquiátrico al discurso público, como clave de lectura de lo social. Hacia 1910, la anhelada profesionalización podía considerarse un hecho; Volker Roelcke (2005) señala que existían 20 escuelas de psiquiatría en las universidades, y que los problemas terminológicos se encontraban mayormente saldados -a partir de la adopción teórica de Kraepelin-. En 1903 se crea la Asociación Alemana de Psiquiatría -que reemplaza a la Asociación de Alienistas existente- y en 1917 se crea en Munich el Instituto Alemán para la Investigación en Psiquiatría (German Research Institute for Psychiatry), bajo la dirección de E. Kraepelin, el cual es incorporado en 1924 al Kaiser Wilhem Institute (KSW), luego rebautizado como Max Plank Institute en la posguerra. Los intereses de hospitales y universidades, ergo, se conjugaban en programas compartidos de investigación y práctica médica.

Por otra parte, no es menor destacar que entre los galenos psi se desarrolló un sensibilidad particular respecto al concepto de Herr Geheimrat, que refería al ideal de un desarrollo profesional que persiguiese la excelencia y la obtención de honores (LÓPEZ-MUÑOZ et al, 2008). Vale señalar aquí que desde fines del siglo XIX y

7 Este viraje y postura propia en los estudios "psi" se reflejó en nombres tales como Wilhelm Griesinger (1817-1868), Emil Kraepelin (1856-1926), Carl Wernicke (1848-1905), y Oswald Bumke (1877-1950), por sólo mencionar algunos casos 


\section{Revista Brasileira de História \& Ciências Sociais - RBHCS}

Vol. $8 \mathrm{~N}^{\circ}$ 16, Julho - Dezembro de 2016

principios del XX los psiquiatras fueron configurando una tríada de principios éticos -explícitos e implícitos- que guiaron su praxis médica, vinculados tanto a la estructura del cuidado de la salud psíquica como a los cambios que el contexto alemán presentaba a fines del XIX, en especial al progresivo reemplazo del concepto de Estado por el de Nación (MOSSE, 2007). Por un lado, entonces, se planteaba la necesidad de estabilizar y restablecer la salud de los individuos; por otra parte aparecía la prosperidad de la economía nacional y, por último, se perseguía la fuerza (biológica) del Estado, objetivo racial que hegemonizó el campo del cuidado de la salud, y especialmente la salud mental.

En el marco del proceso de autonomización del saber psiquiátrico, empero, algunos autores señalan un desplazamiento en las primeras décadas del siglo XX desde el Fürsorge mencionado hacia un enfoque que concebía el cuidado del otro a partir de la prevención global de la sociedad; esto es, hacia un concepto de salud colectiva (Vorsorge) (SEEMAN, 2005). Si bien la atención de la salud ingresó tempranamente en la agenda estatal -junto al enfoque holístico mencionado-, Alemania se configuraba hacia la década del veinte como epicentro del movimiento eugénico a nivel mundial, y la Psiquiatría no fue ajena a sus propuestas, a partir de la articulación de nociones eugénicas junto al concepto de degeneración, entendida como una declinación del status moral, social y de la salud, seguido de un incremento en la carga hereditaria (NOACK, FANGERAU, 2007).

El desarrollo mismo de la somatización del saber psiquiátrico condujo a la creciente popularidad del enfoque negativo de la teoría de la degeneración, que entendía a los desórdenes mentales como una alteración mórbida heredable, y además precisamente por su énfasis sobre el rol atiopatogénico de la herencia y la acción de ciertos agentes tóxicos sobre el organismo- su amplia socialización responde a las posibilidades de control social que dicha "teoría" ofrecía desde la prevención (LÓPEZ-MUÑOZ et al, 2008). ${ }^{8}$ Esta teoría operó, en este sentido, como marco explicativo que permitió amplificar a la psiquiatría por fuera del asilo sobre el

$8 \mathrm{Si}$ bien se registra una primera aproximación en el trabajo de Jacques Joseph Moreau, es con la obra de Bènèdict Augustin Morel -quien en 1857 y 1859 publica sus "Traitè des Dègènèrescences" y su "Traitè des maladies mentales", respectivamente- que el término adquiere connotaciones psiquiátricas. Tanto en la versión moralista de Morel -por su posición católica-como en la reelaboración evolucionista de V. Magnan, la hegemonía del concepto en el campo y el discurso público conllevó un desplazamiento en la importancia del tratamiento del individuo al plano preventivo, insertándose el tratamiento del primero en el marco de un proyecto moralizador al tiempo que estigmatizador de las masas con el fin de preservar al cuerpo social de las consecuencias de sus desviaciones (BING, 2000). 
conjunto del espacio social al habilitar la posibilidad misma de inscribir toda enfermedad posible en la anomalía, decretando la propia inviabilidad del sujeto y estableciendo una herencia indefinida (CAPONI, 2009). 9 De esta manera, una vez que los psiquiatras fueron capaces de vincular la conducta desviada con un cuerpo teórico legitimado fue que pudieron extender su poder por fuera de su praxis tradicional, y que la locura apareció como una tecnología de la anormalidad. ${ }^{10}$

\section{III -Relaciones carnales: Psiquiatría y nazismo}

Como ya dijimos, el discurso psiquiátrico fue ampliamente incorporado en la Alemania del 1900, especialmente por su burguesía intelectual, la cual comenzó a pensar lo social como un sistema biológico, encontrando precisamente en la Eugenesia -positiva o negativa- un método para detener la degeneración (NOACK, FANGERAU, 2007). En este contexto, el programa de la psiquiatría social, de la Eugenesia y la genética psiquiátrica alemanas evidenciaron este cambio en la jerarquía de los valores, tendiendo hacia una mayor preocupación por la detección de conductas "no-sociales" que pudiesen afectar el organismo social (folk-body, Volkskörper). Este movimiento confluyó con el desarrollo general de las ideas eugénicas a nivel europeo y con la corta historia de la higiene mental en Alemania (psychische hygiene), absorbida por un nuevo registro luego de 1933, en clave de higiene racial. ${ }^{11}$ Ya desde las primeras décadas del siglo XX se planteó la "necesidad" de un estudio sistemático de la población, en particular a partir de la influencia de Emil Kraepelin, ${ }^{12}$ quien funda con subsidio estatal el Departamento de Genealogía y

\footnotetext{
$9 \mathrm{Al}$ proponer el concepto de heredo-degeneración y combinar explicaciones anátomopatológicas, sintomáticas y etiológicas-, Magnan generó una primera relectura del concepto, luego retomado e institucionalizado luego por Emil Kraepelin, habilitando una verdadera biologización de las relaciones sociales (CAPONI, 2011).

10 Esta clave de lectura la ofrece Thomas Foth (2013), quien, abordando el desarrollo de terapias convulsivantes por shock durante el nazismo, se posiciona desde el andamiaje teórico de Michel Foucault. Cuando dicha anormalidad se fijó como un status de la herencia individual, sostiene, el proyecto de la cura perdió sentido, desapareciendo así dos cuestiones centrales: el contenido patológico del campo psiquiátrico y su dimensión terapéutica. De esta manera, si lo locos no pueden curarse pero sí ser influenciados por la racionalidad del proyecto psiquiátrico, esta práctica puede definirse como disciplinaria en el sentido foucaultiano: persigue una modificación de la conducta del paciente y se sustenta en una estructura de poder que ubica jerárquicamente al psiquiatra en su cima. 11 Esto marca una clara diferencia con lo acontecido en Francia, donde la Higiene Mental adquirió una primacía mucho más notoria. En términos de orígenes históricos de este viraje, la Sociedad Alemana de Higiene Racial data de 1905 (JOSEPH, WETZEL, 2013).

12 Kraepelin fue uno de los principales partícipes del debate en torno al esquematismo de la Degeneración, apropiándose de la teoría críticamente y dotándola de contenido biológico (BIING, 2000; HOFF, 2008).
} 


\section{Revista Brasileira de História \& Ciências Sociais - RBHCS}

Vol. $8 \mathrm{~N}^{\mathrm{o}}$ 16, Julho - Dezembro de 2016

Demografía en el German Research Institute for Psychiatry, confiado a Ernst Rüdin, su discípulo. A partir de su trabajo, logran instalar una noción devenida en paradigma en el campo psi -“empirical hereditary prognosis”-, al tiempo que, entre 1929-1934, reciben fondos de la Fundación Rockefeller, situación que da cuenta no sólo de su impacto en el desarrollo y la circulación de los saberes especializados a nivel mundial, sino también de la permeabilidad de otros espacios a este tipo de discursos y prácticas de salud (ROELCKE, 2005). El “metadiscurso" de la Eugenesia -y desde ella, la eutanasia- se volvía progresivamente un lente omnicomprensivo desde el cual concebir una serie de políticas de intervención sobre los Otros, cuyos resultados más concretos en los años veinte se observaban también en Estados Unidos (DUDLEY, GALE, 2002), Francia, Gran Bretaña (NOACK, FANGERAU, 2007)13, Dinamarca, Finlandia, Suecia (LÓPEZ-MUÑOZ, 2008) y Canadá (FOTH, 2013).

Durante la República de Weimar, por otro lado, el campo de la Psiquiatría atravesó una diferenciación interna donde surgieron nuevas formas organizacionales de carácter urbano, en pequeña escala e inspiradas por ideales socialistas o psicoanalíticos, las cuales, si bien favorecidas por el gobierno, no contaron con participación de psiquiatras. ${ }^{14}$ La estructura sanitaria asilar incorporó los hospitales privados que fueron surgiendo, mientras se recortaban los recursos públicos para el cuidado psiquiátrico, un panorama en el cual, ante la situación económica general, extendía y cronificaba los períodos de internación, ensanchando la distancia entre población asistida y recursos. Esta situación fue complejizándose y dio lugar a un debate al interior del espectro médico -traducido luego en discurso público- respecto a la asignación diferencial de recursos para estos sectores de la población, llegando a promover procedimientos eutanásicos como solución al problema, idea que el nazismo sólo debería estimular (ROELCKE, 2005).

\footnotetext{
13 Aquí, los motivos para la eliminación física variaban desde un punto de vista eugénico, económico, o desde la perspectiva de la autodeterminación -el propio deseo de morir, muerte como un "acto de misericordia"-.

14 En cuanto a la universidad y la comunidad académica, por lo general fueron opositores a los gobiernos de entreguerras, puesto que los hacían responsables de la pérdida de sus beneficios corporativos, en tanto se consideraban casi como una de las elites del país. Su apoyo al Partido Nazi, en este sentido, fue "recompensado" con la restitución por Hitler de la Ley de Restauración del Personal Civil Profesional, con la reasignación del 55\% del personal universitario (mayormente judío), permitiendo el ingreso y promoción de los simpatizantes (LÓPEZ-MUÑOZ et al, 2008).
} 
A solo seis meses de su acceso a la cancillería, Adolf Hitler sancionó el 14 de julio de 1933 la Ley de Prevención de Procreación Genéticamente Defectuosa, conocida como Sterilization Act. La misma, fruto de los debates médico-raciales suscitados en los años previos (LÓPEZ-MUÑOZ et al, 2007), rigió hasta 1939 y fue aplicada junto a la Ley contra los Criminales Peligrosos, delimitando un amplio margen de intervención sobre los sujetos que constituían un peligro para la raza, contemplando en su lista los “débiles mentales”, esquizofrénicos, alcohólicos, insanos, ciegos, sordos y “deformes” (SEEMAN, 2005). Entre sus principales arquitectos ideológicos tuvo a Ernst Rüdin entonces director del Kaiser Wilhem Institute of Psychiatry de Munich y comisionado de la Sociedad Alemana para la Higiene Mental y Racial15-, cuya obra se articulaba con otras producciones en la misma línea eugénica o eutanásica, como la de Adolf Jost (The right to death, 1895), la profusamente difundida obra de Alfred Hoche y Karl Binding (The permission to destroy Life Unworthy of life, 1920) o los "Principios de la Herencia humana y la Higiene Racial” (1921) de Eugen Fisher, Frtiz Lenz y Erwin Baur -que sirvieron a Hitler como sustento científico para Mein Kampf(NOACK, FANGERAU, 2007). A partir de estas influencias, resulta factible concebir la circulación de nociones específicas que acabaron no sólo por plasmarse en políticas concretas, sino que también se volvieron parte de las representaciones cotidianas de la otredad disruptiva, entre las cuales observamos "ballast lives", "the worthless" o ideas extraídas del Darwinismo Social, Nietzsche, Spengler, la tradición del modernismo reaccionario y un antisemitismo de raíces profundas que acabó por ser medicalizado (LÓPEZ-MUÑOZ et al, 2007).

En igual medida, el advenimiento del Estado nazi modificó de forma considerable la estructura de gobierno del sistema público de salud. Mientras por una parte, generó una centralización tanto del sistema de asilos como de las estadísticas psiquiátricas que estaban en manos de asociaciones disciplinares específicas- bajo un comité de

15 La figura misma de Rüdin resulta un punto de acceso crucial al derrotero de la Psiquiatría alemana del período, en particular por su lugar en la configuración de un campo de estudios en torno a la Higiene Racial y la Psiquiatría Genética (JOSEPH, WETZEL, 2013). En esta dirección, Volker Roelcke (2005) entiende que su trayectoria da cuenta no sólo de la intrincada relación entre genética psiquiátrica, eugenesia y política, sino también del desplazamiento del pluralismo de la República de Weimar a la dominancia de la eugenesia y la higiene racial, situación que no constituyó una ruptura,sino más bien el fortalecimiento de una tendencia ya presente, en detrimento de otra -en particular con la expulsión de sus cultores, de adscripción socialista u origen judío. En efecto, vemos que en 1916 Rüdin ya afirmaba la naturaleza hereditaria de la esquizofrenia -conocida también como demencia precoz en la época-, lo cual implicaba que la misma era refractaria a cualquier intervención terapéutica (POSTEL, 2000). 
directores de asilo y agentes del Estado (German Communal Council), ${ }^{16}$ por otra, redujeron drásticamente los recursos para el tratamiento de los "incurables". En un contexto en el cual hubiese resultado contraproducente emitir una ley explícita de eutanasia, ${ }^{17}$ el nazismo encontró la forma de emprender sus objetivos con leyes como las de 1933, bien con las llamadas Leyes de Nuremberg, promulgadas al año siguiente, y que perseguían la purificación de la sangre del pueblo alemán a partir de la prohibición del casamiento con judíos, entre otras medidas. ${ }^{18}$

La continuación de este proceso se dio con el programa de eutanasia denominado Aktion T4, nombre abreviado de Tiergarten 4, dirección de la inmueble donde funcionaba administrativamente, confiscado a un judío en Berlín. Los antecedentes del mismo provenían de un Decree firmado por el Fuhrer en octubre de 1939, con efectos retroactivos al $1^{\circ}$ de septiembre de dicho año, cuando Alemania invadió Polonia e inició la Segunda Guerra Mundial. Con éste instrumento, el Régimen justificó la muerte de 4000 pacientes psiquiátricos en Polonia, mientras que con la misma lógica se asesinaron 180.00o pacientes psiquiátricos en Alemania entre 19391945 (SEEMAN, 2005). El objetivo del Aktion T4, dirigido por Philip Bouhler y Karl Brandt, era justificar la muerte a gran escala de pacientes hospitalizados y psiquiátricos, a los cuales se incorporaron todas aquellas personas que no cuadraban con el cánon de normalidad, por medio del recurso legal que permitía "brindar" a todo paciente incurable una muerte eutanásica. Para ello, se crearon 21 departamentos que estudiaban los casos clínicos que cada hospital remitía, optando en todos ellos por la muerte del enfermo como solución. Una vez concluida la etapa burocrática, el paciente era enviado a uno de los seis centros regionales que existían

16 Sólo una institución psicoterapéutica "autónoma" continuó existiendo: El Instituto Alemán de Investigación en Psicología y Psicoterapia de Berlin. El mismo sostenía un enfoque ecléctico y distanciado del psicoanálisis, llegando incluso a establecieron una versión propia de la higiene mental, la Psycological Health Guidance. Si bien era marginal en términos cuantitativos -atención de pacientes-, su relevancia (y continuidad) se debía mayormente a la figura de su director, quien era primo del Ministro de Aviación del Reich, agencia a la que prestaron servicios para el personal de la fuerza aérea (ROELCKE, 2005).

17 En efecto, la política oficial de eutanasia se vio influenciada por la experiencia previa del memorandum para una nueva ley criminal nacional-socialista, publicado por el Ministro de Justicia prusiano. Ante las críticas por nacionalismo y racismo, la legalización de la muerte se volvió un tema espinoso para afrontar, a lo cual se sumaba el código publicado por el Ministro de Salud Pública en 1931 que regulaba la experimentación con humanos. Por ello, el régimen persiguió sus fines por distintos medios. En el nivel judicial, se confinó a una ley de esterilización; en el socio-político a cortar el financiamiento a los pacientes psiquiátricos; en el del discurso público y la propaganda, a su estigmatización (LÓPEZ-MUÑOZ et al, 2007).

18 Las esterilizaciones comienzan en 1934 y finalizan con el inicio de la II Guerra, con un total de 350.000 , un $0.5 \%$ de la población, y una tasa de mortalidad que osciló entre el 1 y el $5 \%$ en las prácticas quirúrgicas (ROELCKE, 2005). 


\section{Revista Brasileira de História \& Ciências Sociais - RBHCS}

Vol. $8 \mathrm{~N}^{\mathrm{o}}$ 16, Julho - Dezembro de 2016

para su exterminación física, y que utilizaban como mecanismo principal, desde 1940, duchas para inhalación de monóxido de carbono. ${ }^{19}$ Se crearon, asimismo, departamentos especiales para considerar la situación de los niños con problemas mentales, coordinados por la Cancillería del Furher y al mando de Hanz Heinze, Werner Catel, Ernst Wentzler (NOACK, FANGEREAU, 2007). A partir de una ágil burocratización y del encubrimiento de la muerte de los menores, los casos resueltos terminaban por remitirse a los centros de salud específicos con el objeto de aparentar la aplicación de un tratamiento médico ficticio como forma de justificar ante las familias la muerte del niño en cuestión. Si bien el límite inicial del programa eran los niños de hasta tres años, éste luego se extendió para abarcar a una población cada vez mayor. ${ }^{20}$ En lo referido a la población adulta, existieron dos fases del programa. Entre 1939-1941 funcionó la dependiente del Aktion T4, mientras que entre 19411945 se operó una descentralización, donde se concedió autonomía a los asilos para tomar las decisiones regionalmente, informando de los posibles resultados.

Precisamente, hacia 1941 debieron introducirse algunos cambios en el funcionamiento del programa, sin que por ello se vea afectada la maquinaria de muerte desatada. En tanto algunos de los hospitales psiquiátricos que remitían pacientes o practicaban la eutanasia pertenecían a la Iglesia católica, surgieron fuertes críticas, principalmente en la voz del Arzobispo Clemens Graf Von Galen. Sus repercusiones significaron el final "oficial" y formal del Aktion T4, el cual persistió camuflado bajo otros medios, que algunos autores entendieron como "eutanasia salvaje” (wild eutanasia) (LÓPEZ-MUÑOZ et al, 2007). Estos mecanismos consistían en la propiciación de cuidados deficientes que pudiesen desencadenar nuevas enfermedades en los pacientes, su malnutrición o bien por la aplicación directa tanto de barbituros -que derivaban en casos de neumonía-, así como de inyecciones letales de opiáceos o aire en la sangre. Para la ejecución de esta nueva medida se creó una organización ad hoc, conocida como Operación Brandt, antecedente y campo de experimentación previo a la aplicación de la Solución Final, cuyos métodos de asesinato se perfeccionaron mediante el gas Zyklon B.

En el marco de este conjunto de procesos culturales, que abarcan tanto una construcción radical de la diferencia con el Otro en términos biológico-raciales, hasta

$19 \mathrm{El}$ arquitecto de esta técnica fue Karl Brandt, médico personal de Hitler. (Seeman, 2005).

20 Una descripción concisa del proceso burocrático y concreto de asesinato de los menores en Seeman (2005). 
la banalización y burocratización medicalizada de la muerte -y que, como vimos en el primer apartado, se enmarcan en la progresiva tolerancia a la violencia en Europa luego de la Gran Guerra-, se articulan otras lógicas vinculadas al capitalismo de la "segunda revolución industrial". ${ }^{21}$ En efecto, en las décadas del veinte y el treinta, el desarrollo de la industria farmacológica en Alemania estrechó lazos cada vez más fuertes con la Academia -financiando investigaciones e incorporando a personal en su planta- y el capital financiero, dando lugar al nacimiento del renombrado cartel de la I.G. Farben, un conglomerado de 6 firmas que monopolizó la producción de químicos del país ${ }^{22}$. La proximidad de este grupo empresario con el régimen se manifestó en varios sentidos. No solamente le fue posible absorber muchas empresas de los territorios progresivamente anexados desde 1939, sino que también produjo la totalidad del gas utilizado para la muerte sistemática, desarrolló drogas utilizadas en interrogatorios policiales ${ }^{23}$, participó activamente del programa de armas químicas reactivado desde $1933,{ }^{24}$ y llegó a contar con su propio campo de concentración en Monowitz, vecino al de Auschwitz, donde contaba con libertad de acción. Este anudamiento de intereses resulta, así, una de las claves para comprender el uso sistemático de pacientes como sujetos de experimentación en la investigación farmacológica en Hospitales y universidades, así como de detenidos en los campos de concentración (LÓPEZ-MUÑOZ et al, 2007).25 Sin embargo, algunas figuras de la Academia vinculadas al régimen emprendieron sus propios estudios eugénicos, desarrollados con pacientes vivos, y cuya muerte se premeditaba para realizar luego estudios anátomo-patológicos cerebrales. Carl Schneider, por caso, abordó con sus pacientes ciertas formas de retardo y epilepsia, y junto a Julius Deussen emprendió estudios en histopatología cerebral para comprobar las correlaciones observadas en la

21 Como se señaló en el primer apartado del trabajo, Alemania fue un escenario privilegiado de la misma desde el último tercio del siglo XIX (BARBERO, 2001).

22 Sus principales responsables eran Carl Duisberg (1861-1935) y Carl Bosch (1874-1940), mientras que Hermann Schmitz su arquitecto financiero, mantenía íntimos lazos con el régimen nazi (LÓPEZMUÑOZ et al, 2008).

23 En este sentido merecen destacarse la reutilización de las drogas utilizadas en "curas de sueño" desarrolladas desde los '20 por Jacob Klaesi para el tratamiento de la esquizofrenia, las cuales contaban, en dosis reducidas, con un poder deshinibidor. Este descubrimiento, realizado por William Bleckwenn, fue adoptado por la Gestapo entre sus métodos (LÓPEZ-MUÑOZ, 2008).

24 En 1933 se reactiva el programa de armas químicas, y se emite un decreto que obliga a las empresas a enviar toda información que dispongan y obtengan de químicos desarrollados en el país a la Oficina de Guerra Químicade la Armada, tarea con la que I.G. Farben colabora activamente. Consigue, en efecto, desarrollar potentes neurotóxicos que no llegan a probarse por el final de la guerra, pero que luego los vencedores (y en particular EEUU) perfeccionaron (LÓPEZ-MUÑOZ et al, 2008).

25 La realista y triste metáfora empleada por Enzo Traverso sobre la Guerra Total como laboratorio antropológico donde se gestan las condiciones para los genocidios modernos resulta esclarecedora en este sentido (TRAVERSO, 2003). 


\section{Revista Brasileira de História \& Ciências Sociais - RBHCS}

Vol. $8 \mathrm{~N}^{\mathrm{o}}$ 16, Julho - Dezembro de 2016

fase clínica; Jullius Hallervorden, seleccionaba los cerebros que deseaba estudiar en el centro del Aktion T4 de Bradenburgo, dando su explícita autorización para que sean asesinados por el programa. Pero estos experimentos se desarrollaron también con sujetos sanos extraídos de los campos de concentración: el ejemplo más claro es el de I.G.Farben. (I.G. Auschwitz Industries), que incluso llegó a comprar pacientes clínicamente "relevantes" a otros campos (SEEMAN, 2005).

\section{IV -Cierre}

A lo largo de este trabajo hemos procurado explicitar la compleja relación entre la ciencia médica - particularmente la Psiquiatría- y el advenimiento del nazismo y sus programas de exterminio masivo de poblaciones. Al hacerlo, hemos optado por una estrategia genealógica que permitió inscribir el desarrollo de ciertas nociones y representaciones en suelo alemán como parte de un proceso histórico de larga data, en el cual confluyeron una miríada de elementos. Por una parte, el conjunto de tradiciones y percepciones de los pueblos y espacios que terminaron por constituir Alemania. El carácter explosivo que éstas revestían provenía de las transformaciones socio-políticas y económicas fruto de la unificación, así como por las tensiones surgidas luego del fin de la Gran Guerra y las consecuencias de Versalles. En igual medida, empero, hemos expuesto los derroteros que siguió la medicina germana, la problematización de la Psiquiatría sobre aquello que constituía la enfermedad mental, su distanciamiento respecto a la hegemonía francesa, y el proceso de sedimentación de nuevas significaciones en torno a la degeneración y sus componentes somáticos. Fue, en efecto, el giro producido en Alemania el que permitió a la Psiquiatría en términos globales encontrar bases "científicas" legítimas para su praxis, y de allí su importancia por encima de lo acontecido durante el nazismo.

Ahora bien, la relevancia de este paso en la profesionalización en esta disciplina médica no es sólo una cuestión de esferas de injerencia. El nuevo paradigma kraepeliano, recibido ampliamente por numerosos países que desde allí orientaron sus políticas públicas, fue aplicado en Alemania luego de 1933 en planes sistemáticos de "purificación" racial. Allí reside el punto clave que intentamos elucidar en este trabajo. Esto es, la importancia de rastrear las lógicas impensadas e ingobernables 


\section{Revista Brasileira de História \& Ciências Sociais - RBHCS}

Vol. $8 \mathrm{~N}^{\circ}$ 16, Julho - Dezembro de 2016

que pueden desencadenarse fruto del encuentro entre saberes científicos en una sociedad y un Estado bajo ciertas coyunturas político-culturales.

Si bien es difícil negar la cuota de irracionalidad que caracterizó el accionar del Estado durante la dictadura hitleriana, no es menos cierto que el caudal electoral del partido Nazi observó una escala ascendente en los años previos a su acceso al poder, así como también que el clima intelectual y de la opinión pública alemanes estaban signados por el descontento, la decepción, las consecuencias sociales de la inestabilidad económica, la creciente deslegitimación de un Liberalismo importado, el ascenso de las facciones de derecha y la presencia del comunismo como propuesta societal alternativa. Elementos, todos ellos, procesados desde la particular lente de una cultura volkisch configurada como "modernismo reaccionario", que se diferenciaba de la tradición política liberal del continente, y que era incapaz de metabolizar como propia una cultura política aplicada por imposición, en el marco, además, de una tradición que vinculaba al Estado con la sociedad desde una clave autoritaria.

¿Por qué retomar, entonces, estos aspectos? Porque su importancia es crucial si se pretende alcanzar algún tipo de comprensión sobre el desencadenamiento de ciertas lógicas en ciertos espacios. Ello no implica, desde luego, "comprender" la violencia irracional o la muerte banalizada. Numerosos estudios han abordado ya el problema de los límites de la representación a este respecto. Creemos, sin embargo, que no es un punto menor reposar la atención sobre estas cuestiones puesto que, dada la circulación y recepción de saberes científicos, resulta fundamental que seamos capaces de establecer algunas líneas genealógicas sobre la formación de los mismos. De esta manera, entendemos que es posible observar con otra mirada su apropiación en espacios otros, así como las dinámicas generadas en éstos últimos entre saberes, la sociedad y el Estado. Y más aún cuando ello trata de saberes disciplinares que atañen a la definición de los patrones de lo normal y lo patológico en cada sociedad, y las políticas de exclusión/reclusión diseñadas para ello. 


\section{Bibliografía}

- BARBERO, María Inés. El nacimiento de las sociedades industriales. In ARÓSTEGUI, Julio; BUCHRUCKER, Cristian y SABORIDO, Jorge. El mundo contemporáneo: historia $y$ problemas. Barcelona: Crítica/Biblos, 2001, pp. 67-118.

- BING, Francois. La teoría de la degenerescencia. In, POSTEL, Jacques y QUÉTEL, Claude (coords.). Nueva historia de la Psiquiatría. México: FCE, 2000, p.225-229.

- CAPONI, Sandra. Para una genealogía de la anormalidad: la teoría de la degeneración de Morel. Scientiae Studia, v. 7, nº 3, pp. 425-445, 2011.

- CAPONI, Sandra. Para una genealogía de la psiquiatría ampliada. Cadernos Brasileiros de Saúde Mental, v. 3, nº 6, pp. 106-125, 2009.

- CASALI DE BABOT, Judith; de PRIVITELLO, Luciano. Las revoluciones burguesas y los sistemas políticos del siglo XIX. In ARÓSTEGUI, Julio; BUCHRUCKER, Cristian y SABORIDO, Jorge. El mundo contemporáneo: historia y problemas. Barcelona: Crítica/Biblos, 2001, pp. 135-148.

- DUDLEY, Michel, GALE, Fran. Psychiatrists as a moral community? Psychiatry under the Nazis and its contemporary relevance. Australian and New Zealand Journal of Psychiatry, v. 36, pp. 585-594, 2002.

- FANO, Ester. Los países capitalistas desde la guerra mundial hasta la crisis de 1929. In TELÓ, Mario (coord.). La crisis del capitalismo en los años 20. Análisis económico y debate estratégico en la tercera internacional. Buenos Aires, Ediciones Pasado y Presente, 1981.

- FRITZSCHE, Peter. De alemanes a nazis (1914-1933), Buenos Aires, Siglo XXI, 2009.

- FOTH, Thomas. Shock Therapies as Intensification of the War against Madness in Hamburg, Germany: 1930-1943. Canadian Bulletin of Medical History, v. 30, $\mathrm{n}^{\circ}$ 2, pp. 161-184, 2013

- FOUCAUlt, Michel. El poder psiquiátrico. Curso en el Collége de France 1973-1974, Buenos Aires, Fondo de Cultura Económica, 2012

- FOUCAULT, Michel. Los anormales. Curso en el Collége de France 1974-1975, Buenos Aires, Fondo de Cultura Económica, 2010 
- FOUCAUlT,Michel. Defender la sociedad. Curso en el Collége de France 1975-1976,, Buenos Aires, Fondo de Cultura Económica, 2000

- FOUCAULT, Michel. La vida de los hombres infames, Buenos Aires, Ed Altamira, 1996.

- GAY, Peter. La cultura de Weimar. Una de las épocas más espléndidas de la cultura europea del siglo XIX, Madrid, Paidós, 2011

- HERF, Jeffrey. El modernismo reaccionario. Tecnología, cultura y política en Weimar y el tercer Reich, Buenos Aires, FCE, 1990.

- HOBSBAWM, Eric. Historia del siglo XX, Barcelona, Crítica 1995

- HOFF, Paul. Kraepelin and degeneration theory. European Archives of Psychiatry and Clinical Neurosciences, v. 228, suppl. 2, pp. 12-17, 2008

- JOSEPH, Jay \& WETZEL, Norbert. Ernst Rüdin: Hitler's racial hygene mastermind. Journal of the History of Biology, v. 46, pp. 1-30, 2013.

- KERSHAW, Ian. El Estado Nazi: cun Estado excepcional?. Zona Abierta, ${ }^{\circ}$ 53, pp. 119-148. 1989

- LINZ, Juan J. La crisis de las democracias. In CABRERA, Mercedes, JULIÁ, Santos y ACEÑA, Pedro Martín (comps.). Europa en crisis 1919-1939, Madrid, editorial Pablo Iglesias, 1991, pp. 231-280.

- LÓPEZ-MUÑOZ, Francisco; ÁlAMO, Cecilio; GARCÍA-GARCÍA, Pilar; MOLINA, Juan D.; RUBIO, Gabriel. The role of psychopharmacology in the medical abuses of the Third Reich: From euthanasia programmes to human experimentation. Brain Research Bulletin, v. 77, pp. 388-403, 2008.

- LÓPEZ-MUÑOZ, Francisco; ÁLAMO, Cecilio; DUDLEY, Michael; RUBIO, Gabriel; GARCÍA-GARCÍA, Pilar; MOLINA, Juan D.; OKASHA, Ahmed. Psychiatry and political-institutional abuse from the historical perspective: The ethical lessons of the Nuremberg Trial on their 6oth anniversary. Progress in Neuro-Psychopharmacology \& Biological Psychiatry, v. 31, pp. 791-806, 2007

- MOSSE, George. La nacionalización de las masas. Simbolismo político y movimientos de masas en Alemania desde las guerras napoleónicas al Tercer Reich, Buenos Aires, Siglo XXI, 2007 
- NOACK, Thorsten; FANGERAU, Heiner. Eugenics, Euthanasia, and Aftermath. International Journal of Mental Health, vol. 36, $\mathrm{n}^{\circ}$. 1, pp. $112-124,2007$

- ROELCKE, Volker. Continuities or Ruptures? Concepts, Institutions and Contexts of Twentieth-Century, German Psychiatry and Mental Health Care. In MARIJKE, Gijswijt-Hofstra; OOSTERHUIS, Harry; VIJSELAAR, Joost; FREEMAN, Hugh (Eds.) Psychiatric cultures compared. Psychiatry and Mental Health Care in the Twentieth Century: Comparisons and Approaches, Amsterdam, Amsterdam University Press, 2005, pp. 162182.

- SEEMAN, Mary V. Psychiatry in the Nazi Era. Canadian Journal of Psychiatry, v. 5O, $\mathrm{n}^{\circ}$ 4, pp. 218-225, 2005

- TRAVERSO, Enzo. La historia como campo de batalla. Interpretar las violencias del siglo XX, Buenos Aires, Fondo de Cultura Económica, 2012

- TRAVERSO, Enzo. La sigularidad de Auschwitz. Un debate sobre el uso público de la historia. Cuicuilco, v. 11, $\mathrm{n}^{\circ}$ 31, pp. o-16, 2004.

- TRAVERSO, Enzo. Guerra y memoria. Una mirada sobre el siglo XX desde el presente. Cuadernos del CISH, no 13-14, pp. 215-228, 2003. 